\title{
The object-detection effect: Configuration enhances perception
}

\author{
DEAN G. PURCELL \\ Oakland University, Rochester, Michigan \\ and \\ ALAN L. STEWART \\ Stevens Institute of Technology, Hoboken, New Jersey
}

\begin{abstract}
Line drawings used by Weisstein and Harris (1974) are seen as box-like three-dimensional figures if the lines are arranged properly. A flat two-dimensional pattern is seen when these same lines are disarranged. A target line contained within the three-dimensional figure is identified more readily than is the same line contained within a two-dimensional figure. This finding was extended in the present experiments: The three-dimensional stimulus was detected more quickly than the two-dimensional stimulus, under conditions of visual backward masking. Threedimensional stimuli were also classified more quickly than two-dimensional stimuli. Just as with the face-detection effect and the word-detection effect, object detection can be affected by the form of the visual stimulus.
\end{abstract}

People can quickly pick out a target line that is incorporated within a briefly exposed drawing; they perform best with stimuli that they see as three-dimensional figures. When the same line is part of a drawing that is seen as a flat, two-dimensional pattern, target identification is less accurate (Lanze, Weisstein, \& Harris, 1982; Weisstein \& Harris, 1974; Weisstein, Williams, \& Harris, 1982). This effect is called the object-superiority effect. Examples of three- and two-dimensional patterns are displayed in Figure 1. The lines surrounding the target line form its visual context. There is little doubt that the visual context affects an observer's perception of the target, although it is not obvious how the context influences perception.

Visual context effects are striking. When one looks at Weisstein and Harris's (1974) stimuli, one knows that there is something that needs to be explained, although it is difficult to offer an explanation that is not circular. The difficulty is as follows. One visual context enhances target identification, so the context facilitates perception. By inference, that context must be a more effective visual stimulus. Another arrangement of lines does not facilitate perception. By inference, that context must be a less effective stimulus. Without doubt, a visual context can improve per-

This research was supported by an Oakland University Faculty Research Grant. We thank Michael Botwin for writing the software used in Experiments 1 and 2. We also thank Judy Vindici, Michael Kadar, and Michael Winters for help in data collection. The comments of Lester Krueger, Pierre Jolicoeur, and Eyal Reingold on an earlier draft of this paper are gratefully acknowledged. The data of Experiments 1 and 2 were presented at the 28th Annual Meeting of the Psychonomic Society (Purcell, Stewart, \& Giacoletti, 1987). D. G. Purcell is in the Department of Psychology, Oakland University, Rochester, MI 48309-4401. A. L. Stewart is in the Management Department, Stevens Institute of Technology, Hoboken, NJ 07030. formance. But does the context make the target easier to identify, or does the target line interact with its surrounding context to produce a more effective stimulus?

Other context effects have been demonstrated with subjects who are instructed to report some aspect of a stimulus. Features that form a face can be reported better than features that are disordered to form a jumbled face (Gyoba, Arimura, \& Maruyama, 1980; Homa, Haver, \& Schwartz, 1976; van Santen \& Jonides, 1978). This finding is referred to as the face-superiority effect. Demonstrations of the word-superiority effect show that letters in words are reported better than letters in nonwords (Reicher, 1969; Wheeler, 1970).

We have developed a procedure that escapes even the appearance of circularity and still shows the effect of a visual context on perception. We first used a combination of detection and visual backward masking to study the perception of faces (Purcell \& Stewart, 1981, 1985, 1986, 1987, 1988a, 1988b; Purcell, Stewart, Botwin, \& Kreigh, 1983). The interval separating the onset of the target field from the onset of the masking field is the stimulus onset asynchrony (SOA). Using forced-choice methods, we have shown that a normal face can be detected at an SOA that is shorter than the SOA needed to detect either an inverted or a rearranged face. We refer to this as the facedetection effect. Gorea and Julesz (1990) have reported a face-detection effect with highly schematic faces, but without a pattern mask. An additional demonstration of the effect of meaningfulness on detection showed that, like faces, words are detected better than nonwords (Doyle \& Leach, 1988). We will refer to this effect as the worddetection effect.

People can identify specific features of a stimulus they are familiar with better (face, object, and word superi- 


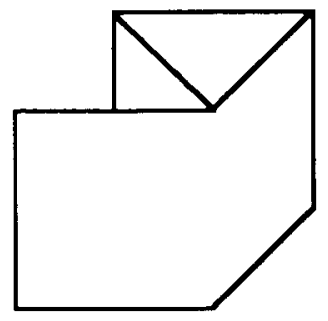

A

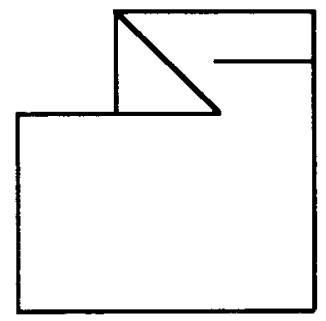

C

Figure 1. Stimuli used in Experiments 1, 2, and 4. Stimulus $A$ was used as the three-dimensional target in Experiments 1, 2, and 4. Stimulus B was used as the two-dimensional stimulus in Experiments 1 and 2. Stimulus $C$ was used as the two-dimensional stimulus in Experiment 4.

ority). They are also faster at detecting a familiar stimulus (face detection, word detection). An additional task has also given evidence of an advantage for familiar stimuli. With visual backward masking, it takes a longer SOA for a subject to classify a face as being normal, inverted, or rearranged, than it takes the subject to detect the corresponding facial stimulus. Normal faces are classified at a shorter SOA than inverted faces are, and inverted faces are classified at a shorter SOA than rearranged facial stimuli are. We (Purcell \& Stewart, 1988a) call this classification advantage for normal faces the face-classification effect. W. R. Uttal and C. Welke (personal communication, 1988) have systematically explored the relationship between face detection, classification, and identification.

The paradigm that we used to demonstrate the facedetection effect can be used with Weisstein and Harris's (1974) stimuli. This paradigm assesses the effectiveness of a stimulus by determining its detection threshold. If the three-dimensional stimuli are more effective than the two-dimensional stimuli, then the three-dimensional stimuli should be detected at a shorter target duration. Our detection technique eliminates the need for a secondary measure of stimulus effectiveness, such as the identification of an arbitrarily chosen line. Instead, it directly assays the perceptibility of objects.

Whatever the cause of the object-superiority effect, it does not produce unqualified superiority. Minor changes in the experimental paradigm can reverse it. Klein (1978) found that the reaction time to identify the slope of the target line was longer for lines embedded within a visual con- text that looked like a three-dimensional object. Pomerantz (1981, p. 178) also found that people took longer to count diagonal lines placed in a three-dimensional context. Similarly, Widmayer and Purcell (1982) found that search time was longer for target lines in a three-dimensional context as opposed to a two-dimensional context. Thus, in tasks in which time to respond is the dependent variable, a threedimensional context does not lead to superior performance, although the stimuli were like those used by Weisstein and Harris (1974). With this in mind, we were uncertain about what we would find as we undertook the following four experiments.

As the results emerged, we discovered that threedimensional objects were usually associated with superior performance, but not always. In Experiment 1, we found that the duration threshold was shorter for detecting a twodimensional stimulus than for detecting a three-dimensional stimulus. However, no masking stimulus followed the onset of the target in Experiment 1. In Experiment 2, when visual backward masking limited the visibility of the stimulus, the detection threshold was shorter for a threedimensional figure, just as we found for the face-detection effect. Experiment 3 confirmed these results, as well as showed that the three-dimensional stimulus had a lower classification threshold than the two-dimensional target did. Like faces, the classification threshold is longer than the detection threshold. In Experiment 4, the stimuli were equated for size (as they were not in Experiment 2) and connectedness (as they were not in Experiment 3). With these additional controls in place, the detection threshold remained lower for the three-dimensional stimulus.

\section{EXPERIMENT 1 \\ Detection Threshold Without Masking}

The subjects in Weisstein and Harris's (1974) experiment looked for a specific line designated as the target. Many investigators have studied how the target line might interact with its context to produce context effects. Factors such as structural relevance (McClelland \& Miller, 1979), emergent features (Pomerantz 1981), target-context redundancy and dissimilarity (Enns \& Prinzmetal, 1984), and fixation point location (Earhard, 1980; Earhard \& Armitage, 1980) are thought to make the target line identifiable. These approaches have missed the point of Weisstein and Harris's (1974) argument, despite their importance for theories of perception.

Weisstein and Harris (1974) argue that the perception of three-dimensional objects is better under some circumstances than the perception of two-dimensional objects is. Weisstein and Harris embedded the target line within the stimulus object. They used the identification of a target line as an indirect measure of the visibility of the entire object. The greater the accuracy of target identification, the more visible the target, and by inference, the more visible the stimulus as a whole.

To study how well an observer can see the three- and two-dimensional stimuli, we turned to the detection procedure used in our work on the face-detection effect (Purcell 
\& Stewart, 1981, 1985, 1986, 1987, 1988a, 1988b; Purcell et al., 1983). Subjects are required only to indicate the location of any change in the visual field in studies of the face-detection effect. The subject does not need to determine what has caused the change in visual field or whether any particular stimulus is used. Any detectable local change in the visual field can alert the observer to the target's presence.

In the discussion of the detectability of a visual stimulus, it is important to state the conditions under which the stimulus is detected. In Experiment 1, we compared the detectability of three- and two-dimensional stimuli when no masking stimulus was present. The dependent variable was the stimulus duration corresponding to the detection threshold. In subsequent experiments, we compared the detectability of the three- and two-dimensional stimuli when they were followed by a patterned masking stimulus. In those experiments, the dependent variable was the SOA that represented the detection threshold under conditions of visual backward masking.

\section{Method}

Subjects. Twenty undergraduate volunteers from introductory psychology classes at Oakland University served as subjects. All had normal or corrected-to-normal vision, and none were aware of the purposes of the experiment.

Apparatus and Stimuli. Two stimuli were used, each derived from the three- and two-dimensional stimuli used by Williams and Weisstein (1984). (See Figure 1.) The three-dimensional context was $1.61^{\circ}$ in width and height. The two-dimensional context had the same height as the three-dimensional context did, but it was $1.09^{\circ}$ wide. The line segments making up the stimuli had a strip width of $0.07^{\circ}$. The target and fixation fields were $9^{\circ}$ wide by $6^{\circ}$ high. A centrally located fixation dot was about $0.07^{\circ}$ in diameter. The stimuli were positioned so that their near edges were $0.73^{\circ}$ to the right or left of the fixation point. The far edge of the threedimensional stimulus was $2.34^{\circ}$ from fixation, while the far edge of the two-dimensional stimulus was $1.82^{\circ}$ from fixation.

Williams and Weisstein (1984) made their three-dimensional figures look quite different from their two-dimensional figures. They were successful in so far as their three-dimensional figures appeared to have great depth, whereas their two-dimensional figures appeared to have little or no depth. Yet, the three- and two-dimensional stimuli differed in two other important ways. The two-dimensional stimuli covered less area than did the three-dimensional stimuli. And the lines composing the two-dimensional stimuli were, on the average, closer to the fixation point than were the lines of the threedimensional stimuli.

A shorter detection threshold for the three-dimensional stimulus might be the result of its perceived depth or its larger size (or a combination of both factors). The detection threshold for the twodimensional stimulus might be briefer because its component lines tended to fall closer to the fixation point than did those of the threedimensional stimulus. Nonetheless, we felt it important to see whether Weisstein and Harris's (1974) stimuli would produce an effect of object detection.

Procedure. The subjects' task was to detect any part of the figure. The target stimulus was positioned to the left or to the right of fixation on each trial. Its position was randomized over trials, as was the type of target presented. Each target was presented an equal number of times in each position. The subjects reported whether the target appeared to the left or to the right of the point of fixation. The subjects were forced to respond on each trial, even if they had to guess. They were informed that they should not try to antic- ipate the side of stimulus presentation, and that they would do best if they simply looked at the fixation dot before starting the trial. The dependent variable was the target duration threshold as determined by the Best PEST algorithm (Lieberman \& Pentland, 1982). Using a two-alternative forced-choice procedure, this corresponds to $75 \%$ correct detection (localization).

Stimuli were presented via a Gerbrands tachistoscope ( 300 series). We adjusted the target field illumination to keep the subjects' thresholds within the range allowed by our equipment. This adjustment was made for each subject during practice $\left(10.28 \mathrm{~cd} / \mathrm{m}^{2}\right.$ for 2 subjects, $4.28 \mathrm{~cd} / \mathrm{m}^{2}$ for 17 subjects, $2.57 \mathrm{~cd} / \mathrm{m}^{2}$ for 1 subject).

The fixation field was constant at $20.56 \mathrm{~cd} / \mathrm{m}^{2}$. The equipment was programmed to present the fixation field for $750 \mathrm{msec}$. This field was replaced by the target field for a duration determined by the threshold assessment algorithm. The fixation field then returned for $750 \mathrm{msec}$. The experiment was run under conditions of normal room illumination in a room that had been painted black (the luminance of the black walls was about $1.7 \mathrm{~cd} / \mathrm{m}^{2}$ ).

The experimenter monitored the experiment, changed the stimulus cards, and keyed the onset of a buzzing sound that indicated to the observer that he or she was free to begin the next trial. Prior to the experiment, the subjects were shown the stimuli outside the tachistoscope. Each subject was then given a block of 16 practice trials with the target duration set to $60 \mathrm{msec}$. This practice block had approximately equal numbers of presentations of the three- and the two-dimensional stimuli. If the subject had any difficulty with the task, the target field luminance was increased and the practice session was rerun. The training was repeated until no errors were made by the subject. Testing took $.5 \mathrm{~h}$. Each threshold value was based on 40 trials. The subjects received a total of 80 experimental trials.

\section{Results and Discussion}

The detection threshold was $17.05 \mathrm{msec}$ for the threedimensional stimulus, and $15.55 \mathrm{msec}$ for the two-dimensional stimulus. A within-subjects analysis of variance (ANOVA) showed that this 1.50 -msec difference in favor of the two-dimensional stimulus was statistically reliable $\left[F(1,19)=5.18, M S_{e}=4.34, p<.05, \eta^{2}=0.21\right]$. We use $\eta^{2}$ as a measure of effect size; it is interpreted as $r^{2}$ (Rosenthal \& Rosnow, 1984) when two conditions are being compared. The results of this experiment are inconsistent with experiments in which line identification was superior with the three-dimensional stimulus (e.g., Weisstein \& Harris, 1974; Williams \& Weisstein, 1984).

Blurring targets, or presenting them off the fovea, reduces the object-superiority effect (Williams \& Weisstein, 1981). Low luminance and short presentations may degrade a stimulus as effectively as blurring it. Certainly, the results of this experiment are consistent with the hypothesis that impoverished stimuli do not produce a robust object-superiority effect. Without a strong objectsuperiority effect, the stimulus that falls closest to the fixation point is the stimulus that is seen best.

\section{EXPERIMENT 2 Detection with Masking}

No object-superiority effect was found in Experiment 1. This result might be trivial. It is possible that we used an inappropriate stimulus presentation. Other superiority effects emerge or disappear as the masking stimulus is changed. The face-detection effect is either absent or 
greatly diminished when target durations are short and the mask is a flash of light (Purcell \& Stewart, 1986). The word-superiority effect also diminishes when a flash mask is substituted for a patterned masking stimulus (Johnston \& McClelland, 1973). Object detection may be similar. The superiority of the three-dimensional stimulus emerges only if its detection threshold is determined under conditions of visual backward masking with a patterned masking stimulus. Consequently, we employed a backward patterned masking stimulus when determining thresholds for two- and three-dimensional stimuli.

\section{Method}

Subjects. Twenty-two undergraduate volunteers served as naive subjects. Each had normal or corrected-to-normal vision. Detection thresholds could not be found for another 5 students who did not serve as subjects.

Apparatus and Stimuli. The apparatus was the same as that in Experiment 1, except that a patterned masking stimulus was used. The mask was composed of a circular array of random bits and pieces of uppercase letters of the alphabet. The white parts of the mask field were set at $20.56 \mathrm{~cd} / \mathrm{m}^{2}$. The space-average luminance of the mask was $10.28 \mathrm{~cd} / \mathrm{m}^{2}$. The white part of the target field was set at $26 \mathrm{~cd} / \mathrm{m}^{2}$.

Procedure. The only change in procedure was the use of a patterned masking stimulus. As in Experiment 1, a 750-msec fixation field preceded the target presentation. At the onset of the target, the fixation field was turned off. The target was exposed for $15 \mathrm{msec}$ and followed by a dark interstimulus interval separating the target offset from the mask onset. Mask duration was fixed at 100 msec. The dependent variable was the SOA that placed a subject at his or her detection (localization) threshold.

\section{Results and Discussion}

The average detection threshold was $\mathrm{SOA}=51.91 \mathrm{msec}$ for the three-dimensional stimulus and SOA $=61.95 \mathrm{msec}$ for the two-dimensional stimulus. A repeated measures analysis of variance showed that this 10.04 -msec difference was statistically reliable $\left[F(1,21)=8.78, M S_{\mathrm{e}}=\right.$ $126.40, p<.01, \eta^{2}=0.295$ ]. This perceptual advantage in favor of the three-dimensional object is similar to the face-detection effect, in which the detection threshold is found at shorter SOA intervals for normal faces than it is for inverted or rearranged faces. Both effects are difficult to produce, unless the target is followed by a patterned masking stimulus (Purcell \& Stewart, 1986). To stress the similarity of the two effects, we will refer to the detection advantage for three-dimensional stimuli as the object-detection effect. The effect size for faces appears larger than that for objects. For normal versus inverted faces, $\eta^{2}=0.53$; for normal versus rearranged faces, $\eta^{2}=0.71$ (Purcell \& Stewart 1988a, Experiment 4).

Detection of the three-dimensional stimulus is better than detection of the two-dimensional stimulus under conditions of visual backward masking, which is consistent with Weisstein and Harris's (1974) finding that target identification is better with a three-dimensional stimulus. Our failure to find an object-detection effect in Experiment 1 was not due to the use of a detection task, but most likely was related to stimulus parameters associated with the lack of a pattern mask.
An ANOVA combining the data of Experiment 1 (no mask) with those of Experiment 2 (mask) allows us to examine the effect of visual masking on stimulus detectability. Experiment (no-mask vs. mask) was the betweensubjects variable. Stimulus dimensionality was the withinsubjects variable. Duration thresholds of Experiment 1 were compared with the SOA thresholds of Experiment 2. These thresholds were reliably different $[F(1,40)=38.98$, $\left.M S_{\mathrm{e}}=353.19, p<.0001\right]$. The main effect of dimensionality was also statistically reliable $[F(1,40)=5.59$, $\left.M S_{\mathrm{e}}=68.42, p<.025\right]$. The critical interaction of experiment $x$ dimensionality was also statistically reliable $\left[F(1,40)=10.20, M S_{\mathrm{e}}=68.42, p<.005\right]$, as it should be if the detection of the three-dimensional stimulus truly changes from inferior performance in Experiment 1 to superior performance in Experiment 2.

The luminance of the stimulus $\left(M=4.8 \mathrm{~cd} / \mathrm{m}^{2}\right)$ was adjusted at the beginning of Experiment 1 for each subject to keep the subject's detection threshold duration within our equipment's range of reliability. This luminance still allowed subjects to detect any stimulus exposed for $60 \mathrm{msec}$ or more. In Experiment 2, the target duration $(15 \mathrm{msec})$ and luminance $\left(26.00 \mathrm{~cd} / \mathrm{m}^{2}\right)$ were chosen to ensure that the target was detectable $100 \%$ of the time only when it was presented without a mask.

Target visibility was probably limited in Experiment 1 because of summation of the fixation luminance with the target (Eriksen, 1966). The duration of the target in Experiment 2 was near the duration thresholds of Experiment 1, but it was tested under high luminance. This combination of duration and luminance was great enough so that an observer in Experiment 2 would easily detect and classify any target not followed by a masking stimulus. The presence of a mask, however, prevented both detection and classification at a sufficiently short SOA, either by interruption of information processing (Turvey, 1973), or by instant-by-instant summation (Stewart \& Purcell, 1974). Either model postulates that processing of the target occurs before the mask; the instant-by-instant model allows limited processing to occur after mask onset.

The targets of Experiments 1 and 2 differed in energy. Like the blurred targets of Williams and Weisstein (1981), the low-luminance rapidly presented targets of Experiment 1 might have generated an indistinct image, even though the precisely tuned mechanism underlying the objectdetection effect needed a characteristic, well-developed image to respond quickly. The higher energy targets of Experiment 2, however, caused sufficient details to emerge quickly enough to engage the object-detection effect mechanism before the masking stimulus could interfere with further processing.

A subject can distinguish the target from the masking pattern if the stimulus precedes the mask by a sufficiently long time. Alternatively, the stimulus must be given a distinctive appearance to allow the subject to segregate the target from the rest of the visual elements of the patterned mask. The stimulus can have its contrast increased to make it distinctive, or, in our case, the stimulus can have a form that is familiar to the observer. But once the features 
coalesce to form a subpattern, the location of the target becomes apparent. The three-dimensional stimuli are patterns that are perceptually prepotent because they are familiar. Their elements coalesce to form a detectable subpattern more quickly than the elements of a twodimensional pattern, which is just what Weisstein and Harris (1974) argued.

\section{EXPERIMENT 3 Detection and Classification}

Our goal was to implement the methods used previously to determine the difference between detection thresholds and classification thresholds for facial stimuli (Purcell \& Stewart, 1987, 1988a; W. R. Uttal \& C. Welke, personal communication, 1988). Our subjects needed only to indicate the spatial or temporal location of the stimulus for detection. For classification however, subjects were required to say whether the face was normal, inverted, or rearranged. In our work on the face-detection effect, we found that detection thresholds (under backward masking) were lower for normal faces than for either inverted or rearranged versions of the same face.

We also found that detection thresholds were lower than classification thresholds. And we found that a subject's ability to classify a face according to type was related to the version of face presented. As with the detection task, there was an advantage for normal faces. However, this face-classification effect was disproportionately larger than the related face-detection effect (Purcell \& Stewart, 1987, 1988a). In other words, the task produced a statistical interaction with face type.

Drawings of objects, presented at their detection threshold, act like faces; subjects detect three-dimensional stimuli more quickly than they detect two-dimensional stimuli. What is not known is whether the classification of objects also parallels the classification of faces. That is, will classification thresholds for objects be higher than detection thresholds, and will object type interact statistically with task, as it does for faces. Faces may be special. Faces may be the only stimuli for which the classification advantage is disproportionately larger than the detection advantage. Such advantages for faces on both detection and classification tasks might be a manifestation of the specialized brain mechanisms used for processing faces (e.g., see Hasselmo, Rolls, Baylis, \& Nalwa, 1989; Perrett, Mistlin, \& Chitty, 1987; Rolls, 1984). It is necessary, therefore, to determine directly if the detection and classification thresholds for three- and two-dimensional stimuli behave as they do for faces.

Object detection should occur before identification, just as detection occurs before identification with other stimuli (Breitmeyer, 1984, p. 133; Dick \& Dick, 1969; Smythe \& Finkel, 1974). Faces and three-dimensional objects might both be thought of as familiar in the sense that both are exemplars of a higher order category. Any normal face is unequivocally and effortlessly judged as such. In the same way, a three-dimensional object is seen as such without recourse to an explicit decision process. If the perceptual familiarity of objects is similar to face type for facial stimuli, then the classification threshold will be lower for the three-dimensional than for the two-dimensional object. Such a finding would establish an important link between object and face processing.

\section{Method}

Subjects. The subjects were 59 undergraduate volunteers from introductory psychology classes at Oakland University. Thirty-three subjects were run with 10 -msec targets, and 26 subjects were run with 5-msec targets. All had normal or corrected-to-normal vision. Two other subjects were not in the sample, because thresholds could not be found for them under at least one of the conditions of the experiment.

Apparatus and Stimuli. All stimuli were presented via an Iconix four-channel tachistoscope. Stimulus presentations were controlled by a Cognitive Testing Station card supplied by Digitry Inc.

Two durations of the target field were tested (5 and $10 \mathrm{msec}$ ). The mask duration was set at 100 msec. The fixation field went on $500 \mathrm{msec}$ before the target onset and went off with the offset of the target. The luminance of all stimulus fields was constant at $48 \mathrm{~cd} / \mathrm{m}^{2}$. A low-luminance $\left(1.28 \mathrm{~cd} / \mathrm{m}^{2}\right)$ fixation field was on continuously. The experiment was carried out in a room that was painted black $\left(\right.$ walls $=1.7 \mathrm{~cd} / \mathrm{m}^{2}$ ).

The stimuli consisted of a perspective drawing of a box, and a drawing of a flat object derived from the constituent parts of the box (see Figure 2). Total line length and line slope were the same for both stimuli. However, intersecting angles and line position differed. Both target stimuli were $2.29^{\circ}$ wide by $2.51^{\circ}$ tall. The stimuli were presented to the left or right of the fixation point. The nearest edge of either stimulus was $0.97^{\circ}$ from the fixation point. The strip width of the line segments was approximately $0.07^{\circ}$.

The flat object was drawn to make it appear as flat as possible. The manner in which it was drawn also affected its connectedness. Although this might confound perceived depth with perceived connectedness, there are two reasons why such a confounding factor is not important. The first reason involves our primary goal in this experiment. We were interested in determining whether it takes a disproportionately longer time to classify a stimulus that is hard to detect. Further, Weisstein et al. (1982) did not find reliable effects of connectedness on the object-superiority effect.

A black $0.07^{\circ}$ dot at the center of the fixation field served as the fixation point. The target, mask, and fixation fields subtended a square $8^{\circ}$ by $8^{\circ}$. The mask was a pattern of overlapping letters $(\mathrm{N}, \mathrm{O})$ that covered the presentation field. Its space average luminance was $24 \mathrm{~cd} / \mathrm{m}^{2}$.

Procedure. The SOA for detection and for classification thresholds was determined with the Best PEST algorithm (Lieberman \& Pent-

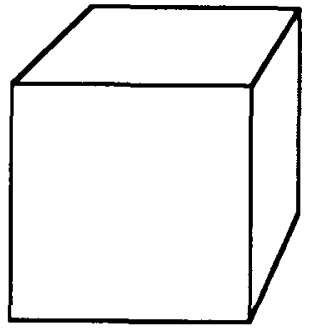

A

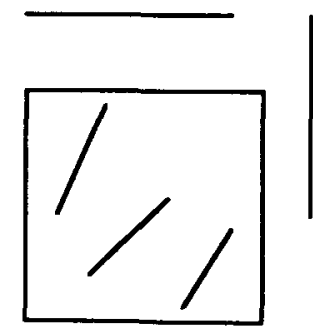

B
Figure 2. The box (A) and flat (B) stimuli used in Experiment 3. 
land, 1982). The smallest step size was $1 \mathrm{msec}$. As in Purcell and Stewart (1988a), there were two types of trials. On detection trials, a response was scored as correct if the subject got the target location correct (left or right of fixation). On classification trials, a response was scored as correct if the subject correctly classified the type of stimulus as box or as flat, and then gave the correct target location (left or right). The subjects were required to make both a classification and a detection response on each trial, as a control for a classification bias (see Purcell \& Stewart, 1988a, p. 360). They responded by saying "box" or "flat," and then saying "left" or "right." As in the previous experiments, the subjects were instructed to make a response on each trial, guessing if necessary.

Calling for both a correct detection and a correct classification response controls for classification bias. At the same time, this requirement introduces the possibility that classification thresholds might be artificially increased over detection thresholds because both responses must be correct. Although this possibility exists in work on the face-detection and face-classification effects (Purcell \& Stewart, 1988a), as well as in Experiment 3, such a bias against classification will not produce the interaction between task and stimulus type found in Purcell and Stewart.

The sequence of target presentation was random, with the constraint that both target types be presented an equal number of times under both tasks. Task was also randomly ordered, with the constraint that both task types be run an equal number of times. The target stimulus was positioned to the right or left of the fixation point at random. The subjects started each trial upon hearing a ready signal generated by the computer. The experimenter monitored the experiment, changed the stimulus cards, keyed the onset of the ready signal, and keyed in each subject's response. The computer scored the responses and gave appropriate feedback with a signal tone.

The subjects were shown the target stimulus cards outside the tachistoscope before the practice session began. They were then given practice in classifying and locating the object at an SOA of $125 \mathrm{msec}$. They were told to give both the target's appearance (box or flat) and its location on each trial. Feedback was given after each trial. During the practice trials, it was emphasized that the subjects were to make both types of responses on each trial. Each subject received $\mathbf{4 0}$ experimental trials for each of the four experimental conditions, for a total of 160 trials. Each session took about $45 \mathrm{~min}$ to complete, with $\mathbf{1 0}$ of those minutes devoted to practice.

A methodological comment. We expected an interaction of task (detect vs. classify) with stimulus dimensionality (three- vs. twodimensional). Therefore, the threshold difference between threeand two-dimensional stimuli should be largest under the classification task. Under both tasks, the three-dimensional stimulus should have the lowest threshold. Our major goal in Experiment 3 was to determine whether task would interact with stimulus dimensionality, as it does when faces are used as stimuli.

A statistical interaction of task with stimulus type is valid only if there are no floor or ceiling effects. The Best PEST algorithm can produce floor effects if the SOA at the detection threshold is close to the target duration. Such floor effects are shown by too few errors in a given condition relative to what is expected from the PEST procedure. An examination of the error scores for each of the conditions of Experiment 3 showed that, with the detection task, the three-dimensional stimulus gave fewer errors than would normally be expected. If floor effects exist in the three-dimensional condition, this would minimize the detection difference between two-dimensional and three-dimensional stimuli while the classification difference is left intact. Such a differential bias between the two tasks might well lead to a statistically reliable interaction without true differences between detection and classification. The conclusion of a parallel between faces and objects would be compromised if the crucial interaction of task $\times$ dimensionality resulted from a floor effect for detection of the three-dimensional stimulus.
With 40 trials per condition, the Best PEST algorithm should produce 10 errors in each condition for the $75 \%$ threshold. Therefore, we set aside the data for each subject with fewer than 8 detection errors for the three-dimensional stimulus. Data from 22 of the original 59 subjects were withdrawn from the sample according to this criterion (18 subjects at a target duration of $10 \mathrm{msec}$, and 4 subjects at a target duration of $5 \mathrm{msec}$ ). More subjects were removed from the 10-msec target condition because the longer target duration was more likely to produce a detection threshold at which the SOA was as short as or shorter than the target duration, producing a floor effect. Removing subjects with floor effects decreases the chance of finding our predicted interaction of task $\times$ condition. However, with these subjects removed from the data set, any interaction of task $\times$ dimensionality is unlikely to be spurious.

\section{Results and Discussion}

Detection and classification. Target duration was a between-subjects variable. Task and stimulus dimensionality were within-subjects variables (see Figure 3). The analysis showed statistically reliable main effects of task $\left[F(1,35)=117.94, M S_{\mathrm{e}}=195.659, p<.0001, \eta^{2}=\right.$ $0.77]$ and dimensionality $\left[F(1,35)=50.98, M S_{\mathrm{e}}=\right.$ 173.391, $\left.p<.0001, \eta^{2}=0.59\right]$. The main effect of target duration was not statistically reliable $[F(1,35)=.154]$. The critical two-way interaction of task $\times$ dimensionality was statistically reliable $\left[F(1,35)=22.87, M S_{\mathrm{e}}=\right.$ $120.61, p<.0001]$. This interaction shows a parallel between objects and faces. The more difficult the target is to detect, the more difficult it is to classify, in consistency with our hypothesis.

The two-way interactions of duration $\times$ task $[F(1,35)=$ $0.18]$ and duration $\times$ dimensionality $[F(1,35)=0.54]$

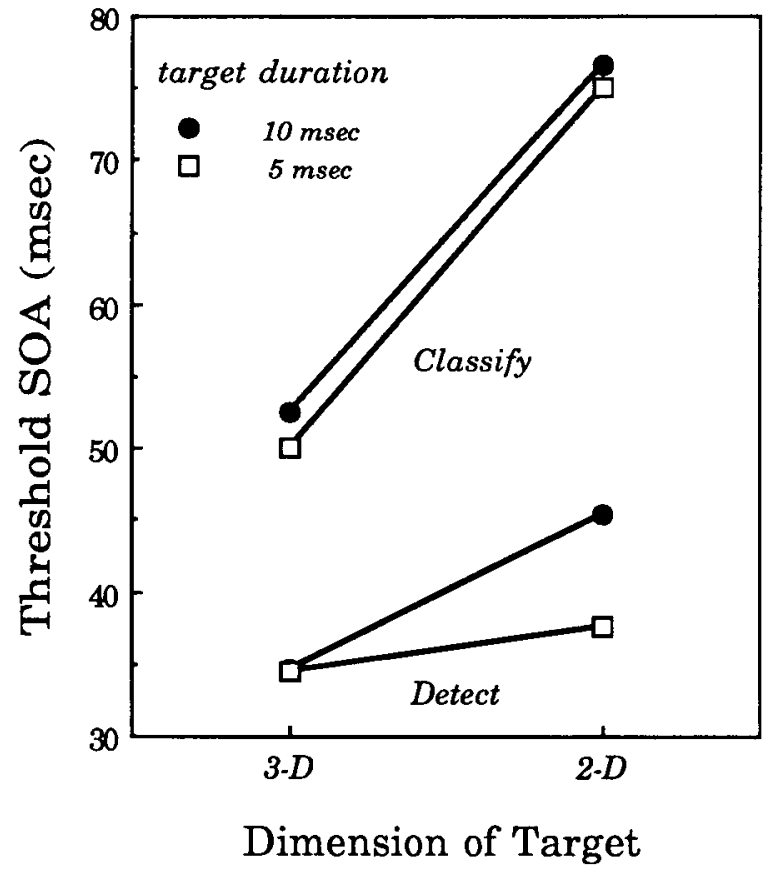

Figure 3. Mean threshold stimulus onset asynchrony for the classification and detection conditions of Experiment 3. Five- and 10-msec target exposures are plotted. 
were not statistically reliable, nor was the three-way interaction of duration $\times$ task $\times$ dimensionality $[F(1,35)=$ $1.30, M S_{\mathrm{e}}=120.61, p=.26 \mathrm{]}$.

For the 10-msec targets, planned comparisons on the detection thresholds for the three-dimensional $(34.73-\mathrm{msec})$ and the two-dimensional $(45.40-\mathrm{msec})$ stimuli showed that there was a statistically reliable object-detection effect of $10.67 \mathrm{msec}\left[F(1,14)=5.96, M S_{\mathrm{e}}=143.19, p<.03\right.$, $\left.\eta^{2}=0.30\right]$. For the 5-msec targets, the thresholds were $34.45 \mathrm{msec}$ for the three-dimensional target and $37.68 \mathrm{msec}$ for the two-dimensional target. This 3.23-msec difference was not statistically reliable $\left[F(1,21)=0.87, M S_{\mathrm{c}}=\right.$ 131.71, $\left.p=.36, \eta^{2}=0.04\right]$.

Both the 5- and the 10-msec presentations produced lower classification thresholds with the three-dimensional stimulus. We will define this difference on the classification task in favor of three-dimensional objects as the object-classification effect to draw attention to its similarity to the face-classification effect (Purcell \& Stewart, 1987, 1988a). For the 10-msec exposures, the threshold for the two-dimensional target $(76.53 \mathrm{msec})$ was $24.06 \mathrm{msec}$ greater than the threshold for the three-dimensional target $(52.47 \mathrm{msec})$. A planned comparison showed that this difference was statistically reliable $\left[F(1,14)=19.18, M S_{\mathrm{e}}=\right.$ $226.53, p<.002, \eta^{2}=0.58$ ]. For the 5-msec targets, the threshold for the two-dimensional target $(75.00 \mathrm{msec})$ was $25 \mathrm{msec}$ greater than the threshold for the threedimensional target $(50.00 \mathrm{msec})$. This difference was also statistically reliable by planned comparison $[F(1,21)=$ $\left.61.50, M S_{e}=111.81, p<.001, \eta^{2}=0.75\right]$. In consistency with the task $\times$ stimulus-type interaction, the effect size was greater for classification than for detection at both target durations.

For the 5-msec targets, the detection difference between three- and two-dimensional targets was not statistically reliable. Eight of the 9 subjects who did not show an objectdetection effect did show an object-classification effect. This effect ( 38.33 vs. $60.67 \mathrm{msec}$ ) was statistically reliable by unplanned comparison test $\left[F(3,8)=12.24, M S_{\mathrm{e}}=\right.$ $61.13, p<.005]$. Their failure to show an object-detection effect may not be due to an insensitivity to three-dimensional objects. It was probably produced by an interaction of the brief duration of the target and the task, or the brief target and the short SOA at which detection occurred.

In the subject sample for Experiment 3, any subject with fewer than eight errors was eliminated from the data set to avoid floor effects. This procedure had the desirable consequence of reducing the likelihood of a spurious interaction of task $x$ dimensionality. A supplemental ANOVA was run as a check on the possibility that subjects with low error scores and, hence, low thresholds might not show the object-detection and object-classification effects. Therefore, this analysis included data from subjects excluded from the primary ANOVA by virtue of their low error scores. Although we do not report the results of this ANOVA here, the pattern of results was the same as that found in the reported ANOVA.
Except for the 5-msec target presentations under the detection task, our conclusion remains intact. Threedimensional stimuli are processed more efficiently than two-dimensional stimuli (Weisstein \& Harris, 1974); they are detected and classified at shorter SOA intervals than two-dimensional stimuli are. The additional time to go from the detection threshold to the classification threshold is reliably less for three-dimensional stimuli than it is for two-dimensional stimuli, a finding that is also consistent with efficient processing of three-dimensional stimuli.

Alternative explanations. Object-superiority effects seem to be associated with enhanced perception, although other explanations suggest themselves. For example, the object-superiority effect might occur because a perceptual property of the stimulus aids its processing (Enns \& Prinzmetal, 1984; Mermelstein, Banks, \& Prinzmetal, 1979; Pomerantz, 1981). The aided-discrimination hypothesis holds that the target lines of a three-dimensional stimulus change the perception of the surrounding context. But this hypothesis does not hold that the stimulus is seen better. Instead, it holds that appropriately constructed three-dimensional stimuli are just easier to distinguish, both from each other and from two-dimensional stimuli.

A related explanation holds that line identification is better when the target line causes a distinctive feature to emerge (Pomerantz, 1981). Different target lines interact with the three-dimensional context to produce objects with different emergent features. A conspicuous emergent feature causes the stimulus to become so distinctive that an observer can easily distinguish it from any other stimulus.

Neither the aided-discrimination nor the emergentfeature explanation applies to our data. Our subjects were required only to locate a three- or a two-dimensional stimulus in space. They did not have to discriminate among a set of stimuli. Any feature that they saw would provide them with enough information so that they could detect the stimulus, regardless of whether the feature was distinctive, emergent, or irrelevant.

A much different explanation of perceptual superiority effects is given by Mermelstein et al. (1979). They argued that visual stimuli are recoded semantically, and that three-dimensional stimuli are more amenable to semantic recoding than two-dimensional stimuli are. The recoded information is then resistant to both visual backward masking and forgetting.

Yet the semantic coding explanation is no more relevant to the object-detection effect than the aided-discrimination hypothesis is. Again, our subjects had only to know where a stimulus was presented in order to detect its presence. But they could detect our stimuli before they could reliably distinguish them as being flat or box-like. Subjects should be able to tell whether the stimuli are flat or boxlike, however, if they have been semantically coded at the time of their detection.

A more general encoding model is appealing, however. Johnston (1981) and Johnston and McClelland (1973) de- 
veloped a retention model of the word-superiority effect. Like the semantic-recoding explanations, the retention model holds that words are represented in a form that is resistant to visual backward masking, although the retention model does not require that words be encoded semantically. Words are retained better than letters, and nonwords, because they are not interfered with by subsequent input. And the longer the word is retained, the more likely attention will shift to the word, bringing it into consciousness.

The idea of a higher order representation that is relatively immune to interference may be useful for our understanding of both object- and face-superiority effects. An advantage should accrue to a stimulus that can be represented in a form resistant to interference from ensuing stimuli. Familiar stimuli, such as faces and objects, may be especially advantaged. Higher order neurophysiological units have been found in the temporal lobes of monkeys (Hasselmo et al., 1989; Perrett et al., 1987; Rolls, 1984). Such representation cannot be semantic for Rhesus monkeys, and need not be semantic for human observers. Similar units may exist for objects. If a stimulus has features that activate such units, that stimulus will be encoded more rapidly and hence detected more quickly.

Experiments 2, 3, and 4 provide direct proof that the three-dimensional stimulus is a more effective stimulus than the two-dimensional stimulus under conditions of visual backward masking by a patterned stimulus. This finding is also consistent with Weisstein and Harris's (1974) interpretation of their data. We have circumvented the problem of confusing cause with effect, however. Before the present experiments, there was always the question of whether it was the context that made the target easier to identify or the target line interacting with its local environment that facilitated identification. The problem is now easier to recognize. The target and the context are fused when they form a familiar pattern.

\section{EXPERIMENT 4 \\ Size and Connectedness Control}

Experiment 4 was designed in response to the criticism of an anonymous reviewer. The two-dimensional stimulus of Experiments 1 and 2 was taken from Williams and Weisstein (1984). Just as in their study, our two-dimensional stimulus covered a smaller area than the three-dimensional stimulus did. It did, however, contain the same total length of line. Although a difference in stimulus area should not influence target line identification (the task used by Weisstein \& Harris, 1974), it might have an effect on target detection. Although the reversal of the objectdetection effect found in Experiment 1 mitigates a stimulus area hypothesis, the differences in size might make the three-dimensional stimulus more visible under conditions of backward masking.

\section{Method}

Subjects. The subjects were 21 undergraduate volunteers from introductory psychology classes at Oakland University. Three other subjects were not in the sample, because of a failure to achieve a stable threshold in one or more conditions.

Stimuli and Apparatus. The three-dimensional stimulus was the same as in Experiments 1 and 2. The two-dimensional stimulus was modified to cover about the same area as that covered by the threedimensional stimulus (see Figure 1). Although total line length was the same for both stimuli, the two-dimensional stimulus covered slightly more area than the three-dimensional stimulus did. Both stimuli subtended $1.57^{\circ}$ in height and width. They were presented so that the closest contour fell $0.78^{\circ}$ from the fixation point. The strip width of line segments from which the stimuli were constructed was about $0.05^{\circ}$.

The apparatus and conditions of stimulus presentation were the same as in Experiment 3, with the following exceptions. The target duration was adjusted individually for each subject to keep detection thresholds within the operational limits of our equipment (10 msec for 10 subjects; 5,15 , and 20 msec for 3 subjects each; 25 and $30 \mathrm{msec}$ for 1 subject each). Only the detection threshold was determined. The luminance of the white background of the target and fixation fields was $51.39 \mathrm{~cd} / \mathrm{m}^{2}$. The white background of the mask field was set at $39.39 \mathrm{~cd} / \mathrm{m}^{2}$.

Procedure. The procedure was the same as that in Experiment 2. The subjects were shown the target stimulus cards outside the tachistoscope before the practice session began. They were then given practice in locating the object, at an SOA of $125 \mathrm{msec}$. Each subject received 40 experimental trials for each of the two experimental conditions, for a total of 80 trials. Each session took about $30 \mathrm{~min}$ to complete, with 10 of those minutes devoted to practice.

\section{Results and Discussion}

The SOA detection thresholds were $48.90 \mathrm{msec}$ for the three-dimensional stimulus and $54.29 \mathrm{msec}$ for the two-dimensional stimulus. A repeated measures ANOVA showed that this $5.39-\mathrm{msec}$ difference, in favor of the three-dimensional stimulus, was statistically reliable $\left[F(1,20)=4.62, M S_{e}=65.774, p<.05, \eta^{2}=0.19\right]$. Thus, the object-detection effect is found with stimuli that are of the type used in Experiments 1 and 2, but that do not confound size or connectivity with dimensionality.

Experiment 4 was designed to eliminate the confounding of stimulus area with the type of stimulus (three- and two-dimensional). If the area of the two-dimensional stimulus affects the size of the object-detection effect, then that effect should have been smaller in Experiment 4, in which the differences in size were small. This was indeed the case. The magnitude of the difference between threeand two-dimensional stimuli was larger for Experiment 2 $\left(10.04 \mathrm{msec}, \eta^{2}=0.30\right)$ than for Experiment $4(5.39 \mathrm{msec}$, $\left.\eta^{2}=0.19\right)$. However, a between-subjects ANOVA revealed that this difference was not reliable $[F(1,41)=$ $\left.1.21, M S_{\mathrm{e}}=193.66, p=.28, \eta^{2}=0.03\right]$. A more powerful within-subjects experiment might well yield a reliable difference in the size of the object-detection effect under the stimulus conditions of Experiments 2 and 4. What is clear from the data of Experiment 4 is that the object-detection effect as found in Experiment 2 is not solely due to two-dimensional stimulus size.

The size of the object-detection effect was larger for the 10-msec targets of Experiment $3\left(10.67 \mathrm{msec}, \eta^{2}=\right.$ $0.30)$ as opposed to Experiment $4\left(5.38 \mathrm{msec}, \eta^{2}=\right.$ $0.19)$. Although this is consistent with the argument that connectivity affected the size of the object-detection ef- 
fect, this difference was not statistically reliable $[F(1,34)=$ $\left.1.252, M S_{e}=195.30, p=.27, \eta^{2}=.04\right]$. Care should be taken when one interprets the differences between Experiment 3 and Experiment 4, because the stimuli differed in size and dimensionality, as well as connectedness.

\section{GENERAL DISCUSSION}

Familiar perceptual organization enhances detectabilityat least, this is what we believe to be the significance of the face-detection and object-detection effects. The advantage given to faces and three-dimensional objects may emerge from the fact that they can be organized spontaneously by the visual system. A familiar stimulus is likely to be further along in its visual processing at any time after its onset than a less familiar stimulus is. Speaking loosely, the observer has a better look at a briefly presented stimulus if it is familiar. Just as with faces, people perform better with three-dimensional stimuli because they are more familiar and therefore are seen better at short values of SOA (Purcell \& Stewart, 1986, 1988a).

The fact that people see three-dimensional stimuli better than two-dimensional stimuli may also account for objectinferiority effects. When stimuli are presented for longer periods of time and the dependent variable is the time to find a specified target, an object-inferiority effect is found (Klein, 1978; Pomerantz, 1981, p. 178; Widmayer \& Purcell, 1982). Indeed, when object inferiority is found, the target exposure times are 10-100 times those with which one finds the object-detection and object-superiority effects. If we call for a subject to tell us something specific about a subfeature of a stimulus, the organization (or familiarity) of the pattern may interfere with an observer's ability to locate the subfeature (Mermelstein et al., 1979; Pomerantz, 1981). To identify a particular line, the observer must decompose the figure mentally in his or her search to find the target item, or make some other judgment about a particular part of a figure. We know that targets that are parts of a gestalt are difficult to identify (Prinzmetal \& Banks, 1977). In much the same way, the more familiar a figure, the more difficult it is to find a target item that is part of the figure. Hence, the properties of a stimulus that make it more detectable may be the same properties that make its mental decomposition most difficult. Instead of presenting a paradox, the inferiority or the superiority of a stimulus are part and parcel of what makes it detectable in the first place.

In discussing the face-detection effect, we suggested that a face, as an organized, familiar pattern, is a more potent stimulus than an arbitrary assemblage of the same visual features (Purcell \& Stewart, 1988a). In the facedetection effect, the detectability of the stimulus is caused by more than just the physical intensity and distribution of light. An upright face is physically identical to an inverted face, and both an upright face and an inverted face stimulate the same area of good and poor acuity. It seems, therefore, that in addition to its physical parameters of size, position, and pattern of luminance, it is the familiar- ity of the stimulus for the observer that makes a face more detectable when it is upright. By extension, rearranging facial stimuli makes the pattern less familiar and therefore less detectable.

This same phenomenon seems to hold for words (Doyle \& Leach, 1988) and for Weisstein and Harris's (1974) figures. Some arrangements of letters are more familiar than others, just as some arrangements of lines result in pictures that are more familiar for the viewer. The more familiar the arrangement is for the observer, the more detectable the stimulus. The face-detection effect, the worddetection effect, and the object-detection effect all share the property that detectability is determined by familiarity. It seems, therefore, that there is a class of informationdependent detection effects. The form of the representation of this information has yet to be determined.

\section{REFERENCES}

BreitMeyer, B. G. (1984). Visual masking: An integrative approach. New York: Oxford University Press.

Dick, A. O., \& Dick, S. O. (1969). An analysis of hierarchical processing in visual perception. Canadian Joumal of Psychology, 23, 203-211.

DOYLE, J. R., \& LEACH, C. (1988). Word superiority in signal detection: Barely a glimpse, yet reading nonetheless. Cognitive Psychology, 20, 283-318.

EARHARD, B. (1980). The line-in-object superiority effect in perception: It depends on where you fix your eyes and what is located at the point of fixation. Perception \& Psychophysics, 28, 9-18.

EARHARD, B., ARMITAGE, R. (1980). From an object-superiority effect to an object-inferiority effect with movement of the fixation point. Perception \& Psychophysics, 28, 369-376.

EnNs, J. T., Prinzmetal, W. (1984). The role of redundancy in the object-line effect. Perception \& Psychophysics, 35, 22-32.

ErIKSEN, C. W. (1966). Temporal luminance summation effects in backward and forward masking. Perception \& Psychophysics, 1, 87-92.

Gorea, A., Julesz, B. (1990). Context superiority in a detection task with line-element stimuli: A low-level effect. Perception, 19, 5-16.

Gyoba, J., Arimura, M., \& Maruyama, K. (1980). Visual identification of line segments embedded in human face patterns. Tohoku Psychologica Folia, 39, 113-120.

Hasselmo, M. E., Rolls, E. T., Baylis, G. C., \& Nalwa, V. (1989). Object-centered encoding by face-selective neurons in the cortex in the superior temporal sulcus of the monkey. Experimental Brain Research, 75, 417-429.

Homa, D., Haver, B., Schwartz, T. (1976). Perceptibility of schematic face stimuli: Evidence for a perceptual Gestalt. Memory \& Cognition, 4, 176-185.

Johnston, J. C. (1981). Understanding word perception: Clues from studying the word-superiority effect. In $\mathrm{O}$. J. L. Tzeng \& H. Singer (Eds.), Perception of print: Reading research in experimental psychology (pp. 65-84). Hillsdale, NJ: Erlbaum.

JoHNSTON, J. C., \& MCCLELLAND, J. L. (1973). Visual factors in word perception. Perception \& Psychophysics, 14, 365-370.

KLEIN, R. (1978). Visual detection of line segments: Two exceptions to the object superiority effect. Perception \& Psychophysics, 24, 237-242.

Lanze, M., Weisstein, N., Harris, J. R. (1982). Perceived depth vs. structural relevance in the object-superiority effect. Perception \& Psychophysics, 31, 376-382.

Lieberman, H. R., Pentland, A. P. (1982). Microcomputer-based estimation of psychophysical thresholds: The Best PEST. Behavior Research Methods \& Instrumentation, 14, 21-25.

McClelland, J. L., \& Miller, J. (1979). Structural factors in figure perception. Perception \& Psychophysics, 26, 221-229.

Mermelstein, R., Banks, W., Prinzmetal, W. (1979). Figural 
goodness effects in perception and memory. Perception \& Psychophysics, 26, 472-480.

Perrett, D. I., Mistlin, A. J., \& Chitty, A. J. (1987). Visual neurones responsive to faces. Trends in Neuroscience, 10, 358-364.

Pomerantz, J. R. (1981). Perceptual organization. In M. Kubovoy \& J. R. Pomerantz (Eds.), Perceptual organization (pp. 141-180). Hillsdale, NJ: Erlbaum.

Prinzmetal, W., \& Banks, W. P. (1977). Good continuation affects visual detection. Perception \& Psychophysics, 21, 389-395.

Purcell, D. G., Stewart, A. L. (1981, November). A face superiority effect. Paper presented at the meeting of the Psychonomic Society, Philadelphia.

Purcell, D. G., Stewart, A. L. (1985, November). The face detection effect. Paper presented at the meeting of the Psychonomic Society, Boston.

Purcell, D. G., \& Stewart, A. L. (1986). The face-detection effect. Bulletin of the Psychonomic Society, 24, 118-120.

Purcell, D. G., \& Stewart, A. L. (1987, August). Face detection and identification. Paper presented at the meeting of the American Psychological Association, New York.

Purcell, D. G., Stewart, A. L. (1988a). The face-detection effect: Configuration enhances detection. Perception \& Psychophysics, 43, 355-366.

Purcell, D. G., \& Stewart, A. L. (1988b, November). The face detection effect: Effects of positional uncertainty and contrast. Paper presented at the meeting of the Psychonomic Society, Chicago.

Purcell, D. G., Stewart, A. L., Botwin, M., \& Kreigh, R. (1983, November). A face superiority effect: Hemiretina effects. Paper presented at the meeting of the Psychonomic Society, San Diego.

Purcell, D. G., Stewart, A. L., \& Giacoletti, A. M. (1987, November). The object detection effect. Paper presented at the meeting of the Psychonomic Society, Seattle.

ReICHER, G. M. (1969). Perceptual recognition as a function of meaningfulness of stimulus materials. Joumal of Experimental Psychology, 81, 275-280.
RoLLs, E. T. (1984). Neurons in the cortex of the temporal lobe and in the amygdala of the monkey with responses selective for faces Human Neurobiology, 3, 209-222.

RosenthaL, R., \& RosNow, R. L. (1984). Essentials of behavioral research. New York: McGraw-Hill.

SMYTHE, L., \& FINKEL, D. L. (1974). Masking of spatial and identity information from geometric forms by a visual noise field. Canadian Journal of Psychology, 28, 399-408.

Stewart, A. L., \& PurCell, D. G. (1974). Visual backward masking by a flash of light: A study of U-shaped detection functions. Journal of Experimental Psychology, 103, 553-566.

TURVEY, M. T. (1973). On peripheral and central processes in vision: Inferences from an information-processing analysis of masking with patterned stimuli. Psychological Review, 80, 1-52.

Van SANTEN, J. P. H., \& Jonides, J. (1978). A replication of the facesuperiority effect. Bulletin of the Psychonomic Society, 12, 378-380.

WeIsstein, N., \& HaRRIS, C. S. (1974). Visual detection of line segments: An object-superiority effect. Science, 186, 752-755.

Weisstein, N., Willams, M. C., \& Harris, C. S. (1982). Depth, connectedness, and structural relevance in the object-superiority effect: Line segments are harder to see in flatter patterns. Perception, 11, 5-17.

Wheeler, D. D. (1970). Processes in word recognition. Cognitive Psychology, 1, 59-85.

Widmayer, M., \& Purcell, D. G. (1982). Visual scanning of line segments: Object superiority and its reversal. Bulletin of the Psychonomic Society, 19, 353-354.

Williams, M. C., \& Weisstein, N. (1981). Spatial frequency response and perceived depth in the time-course of object superiority. Vision Research, 21, 631-646.

Williams, M. C., \&eisstein, N. (1984). The effect of perceived depth and connectedness on metacontrast functions. Vision Research, 24, 1279-1288.

(Manuscript received November 15, 1989; revision accepted for publication March 12, 1991.) 